\title{
Downregulation of microRNA-301a inhibited proliferation, migration and invasion of non-small cell lung cancer by directly targeting DLC1
}

\author{
ZHUYU WU ${ }^{1,2}$, YAOJUN LI ${ }^{2}$ and GUOJUN ZHANG ${ }^{1}$ \\ ${ }^{1}$ Department of Respiratory Medicine, The First Affiliated Hospital of Zhengzhou University, Zhengzhou, \\ Henan 450000; ${ }^{2}$ Department of Respiratory Medicine, Luohe Central Hospital, Luohe, Henan 462000, P.R. China
}

Received March 3, 2016; Accepted August 1, 2017

DOI: $10.3892 / \mathrm{ol} .2017 .6990$

\begin{abstract}
Increasing evidence has indicated that the abnormal expression of microRNAs contributes to tumorigenesis and tumor development. Understanding the roles of microRNAs in non-small cell lung cancer (NSCLC) might provide valuable information for therapeutic strategies in the therapy for patients with NSCLC. In the present study, significant upregulation of microRNA (miR)-301a was observed in NSCLC tissues and cell lines compared with normal adjacent tissues and a normal human bronchial epithelial cell line. The inhibition of miR-301a suppressed proliferation, migration and invasion of NSCLC cells. Functional analyses indicated that DLC1 was a direct target of miR-301a in NSCLC. Inhibiting miR-301a expression decreased DLC1 expression at mRNA and protein levels. Moreover, DLC1 knockdown partially reversed the inhibition of proliferation, migration and invasion induced by miR-301a knockdown in NSCLC cells. Therefore, these findings may provide novel insights into the molecular mechanisms of miR-301a in proliferation, migration and invasion of NSCLC cells. The findings also indicated that miR-301a may act as a novel potential therapeutic target for patients with NSCLC.
\end{abstract}

\section{Introduction}

Lung cancer is one of the most common types of cancer and is the leading cause of cancer-associated mortality worldwide, with an estimated of $>200,000$ new cases every year (1). Non-small cell lung cancer (NSCLC), the most common type of lung cancer, which accounts for $85 \%$ of all lung cancer cases, includes four subtypes: Adenocarcinoma, squamous

Correspondence to: Professor Guojun Zhang, Department of Respiratory Medicine, The First Affiliated Hospital of Zhengzhou University, 1 Jianshe East Road, Zhengzhou, Henan 450000, P.R. China

E-mail: guojunzhang_zzu@163.com

Key words: miRNA-301a, NSCLC, lung cancer, proliferation, migration, invasion, DLC1 cell carcinoma, adenosquamous cell carcinoma and large cell carcinoma (2). In China, the incidence of NSCLC is still increasing in urban and rural areas (3). For NSCLC patients with early-stage or locally advanced lung cancer, surgery is the most effective treatment, followed by chemotherapy and radiotherapy. For stage III/IV NSCLC, platinum-based combined chemotherapy is the current standard of treatment $(4,5)$. In recent years, great advancement has been made in the management of NSCLC. However, the prognosis of NSCLC remains unfavorable, with a 5-year overall survival rate of $\sim 16 \%(6,7)$. Therefore, fully understanding of the molecular mechanisms underlying NSCLC carcinogenesis and progression is necessary for improving the diagnosis, prevention and treatment of patients with NSCLC.

MicroRNAs (miRNAs) are a large family of small, non-protein-coding and single-stranded RNA molecules that range from 19 to 25 nucleotides in length (8). miRNAs have important roles in gene expression through interacting with complementary nucleotide sequences in the $3^{\prime}$ untranslated regions (3'UTRs) of target mRNAs, leading to degradation or translational suppression of their target mRNAs (9). A single miRNA can regulate the expression of a wide variety of target genes, which contain target binding sites that interact with miRNAs. Additionally, a single mRNA can be regulated by a number of different miRNAs (10). More than $60 \%$ of human genes have been predicted to be regulated by miRNAs, and therefore miRNAs are involved in various biological processes, including development, cell proliferation, cell cycle, apoptosis, differentiation, metastasis and resistance to chemotherapeutics (11-13). An increasing number of studies reported that miRNAs are frequently dysregulated in a large number of types of human cancer and have important roles in the regulation of carcinogenesis and disease progression $(9,14)$. Certain miRNA may act as an oncogene or a tumor suppressor gene, depending on the roles of their target genes (15). Based on these findings, miRNAs may be investigated as a therapeutic targets in cancer diagnosis, prevention and therapy.

In the present study, the expression levels of miR-301a in NSCLC tissues and cell lines were investigated, and its roles on NSCLC cell proliferation, migration and invasion were evaluated. The results of the present study will provide further insights into the molecular mechanisms responsible for NSCLC 
carcinogenesis and progression, and identify miR-301a as a potential therapeutic target in NSCLC treatment.

\section{Materials and methods}

Clinical samples. A total of forty-two paired NSCLC tissues and adjacent non-tumor lung tissues were obtained from patients (29 males, 13 females; age range, 47-79 years; mean age, 58 years) receiving surgical treatment at The First Affiliated Hospital of Zhengzhou University between February 2012 and August 2014. All patients did not receive chemotherapy or radiotherapy prior to surgery. All tissues were excised, immediately snap-frozen in liquid nitrogen and then transferred to $-80^{\circ} \mathrm{C}$ refrigerator until use. The present study was approved by the Ethical Committee of The First Affiliated Hospital of Zhengzhou University (Zhengzhou, China), and informed consent was obtained from all patients.

Cell culture and transfection. A total of five NSCLC cell lines (NCI-H1975, H1299, SPC-A-1, H520 and A549), a normal human bronchial epithelial cell line (16HBE) and 293 were purchased from Institute of Biochemistry and Cell Biology of the Chinese Academy of Sciences (Shanghai, China). These cell lines were cultured in Dulbecco's modified Eagle's medium (DMEM; Gibco; Thermo Fisher Scientific, Inc., Waltham, MA, USA) or RPMI 1640 medium (Gibco; Thermo Fisher Scientific, Inc.) supplemented with $10 \%$ fetal bovine serum (FBS; Gibco; Thermo Fisher Scientific, Inc.), $2 \mu \mathrm{M}$ glutamine (Gibco; Thermo Fisher Scientific, Inc.), as well as $100 \mathrm{U} / \mathrm{ml}$ penicillin and $100 \mu \mathrm{g} / \mathrm{ml}$ streptomycin (Gibco; Thermo Fisher Scientific, Inc.). All cells were kept in a humidified incubator at $37^{\circ} \mathrm{C}$ with $5 \% \mathrm{CO}_{2}$.

The miR-301a inhibitor, negative control (NC) inhibitor, DLC1 small-interfering RNA targeting DLC1 (DLC1 siRNA) and NC siRNA were purchased from Shanghai GenePharma Co., Ltd., (Shanghai, China). The DLC1 siRNA sequence was 5'-TACGTCCAAGGTCGGGCAGGAAGA-3' and the NC siRNA sequence was 5'-UUCUCCGAACGUGUCACG UTT-3'. H1299 and A549 cells were seeded in six-well plates to $70 \%$ confluence and transfected with miRNA inhibitor (100 pmol) or siRNAs (100 pmol) using Lipofectamine 2000 (Invitrogen; Thermo Fisher Scientific, Inc.) according to the manufacturer's instructions. This assay did not use untransfected cells. After transfection $48 \mathrm{~h}$, RT-qPCR was performed to detect miR-301a expression. CCK-8 and cell migration and invasion assays were carried out at 24 and $48 \mathrm{~h}$ following transfection. Western blotting analysis was carried out at $72 \mathrm{~h}$ following transfection.

Reverse transcription-quantitative polymerase chain reaction $(R T-q P C R)$. Total RNA from tissues and cells (H1299 and A549) was isolated using TRIzol reagent (Invitrogen; Thermo Fisher Scientific, Inc.) according to the manufacturer's protocols. For miR-301a expression, RT-qPCR was performed using the SYBR ${ }^{\circledR}$ Green PCR kit (Toyobo Life Science, Osaka, Japan) on an Applied Biosystems Realtime 7500 Sequence Detection system (Applied Biosystems; Thermo Fisher Scientific Inc.). The primer sequences for miR-301a and U6 were as follows: miR-301a forward, 5'-ACACTCCAGCTGGGCAGTGCA ATAGTATTGTC-3' and reverse, 5'-CTCAACTGGTGTCGT
GGA-3'; and U6 forward, 5'-GCTTCGGCAGCACATATA CTAAAAT-3' and reverse, 5'-CGCTTCACGAATTTGCGT GTCAT-3'. For DLC1 mRNA detection, reverse transcription was performed using the PrimeScript RT reagent kit (Takara Biotechnology Co., Ltd., Dalian, China) followed by qPCR using SYBR Premix Ex Taq (Takara Biotechnology Co., Ltd.). The primer sequences were as follows: DLC1 forward 5'-CCG CCTGAGCATCTACGA-3' and reverse, 5'-TTCTCCGAC CACTGATTGACTA-3'; $\beta$-actin forward, 5'-CCTGGCACC CAGCACAAT-3' and reverse, 5'-GCTGATCCACATCTG CTGGAA-3'; miR-301a forward, 5'-ACACTCCAGCTGGGC AGTGCAATAGTATTGTC-3' and reverse, 5'-CTCAACTGG TGTCGTGGA-3'; and U6 forward, 5'-GCTTCGGCAGCA CATATACTAAAAT-3' and reverse, 5'-CGCTTCACGAAT TTGCGTGTCAT-3'. miR-301a expression was normalized to U6 expression, and $\beta$-actin was used as an internal control for DLC1 mRNA expression. The relative expression was analyzed with the $2^{-\Delta \Delta \mathrm{Cq}}$ method (16).

Cell counting Kit-8 (CCK-8) assay. To evaluate the effects of miR-301a on the proliferation of NSCLC cells, the CCK8 kit (Dojindo Molecular Technologies, Inc., Kumamoto, Japan) was used. For this assay, transfected cells were collected and seeded into 96-well plates at an initial density of 3,000 cells/well. Cell proliferation was detected every $24 \mathrm{~h}$ until $96 \mathrm{~h}$ according to the manufacturer's protocol. At each time points, $10 \mu \mathrm{l} \mathrm{CCK8}$ solution was added to each well and cultured for $2 \mathrm{~h}$ at $37^{\circ} \mathrm{C}$. Optical density (OD) was measured at a wavelength of $450 \mathrm{~nm}$ using a microplate reader. Each experiment was performed in three replicates and repeated three times.

Cell migration and invasion assay. To assess the effects of miR-301a on migration and invasion of NSCLC cells, cell migration and invasion assays were performed using 24-well Transwell chambers ( $8 \mathrm{~mm}$ pore size; BD Biosciences, Franklin Lakes, NJ, USA). For cell migration assays, transfected cells were collected at $48 \mathrm{~h}$ post-transfection, suspended into FBS-free culture medium and seeded in the upper chambers at an initial density of $5 \times 10^{4}$ cells/well. The lower chambers were filled with $500 \mu \mathrm{l}$ DMEM containing $20 \% \mathrm{FBS}$ as a chemoattractant. Following incubation at $37^{\circ} \mathrm{C}$ for $48 \mathrm{~h}$, the non-migrated cells were carefully removed with a cotton swab. The migrated cells were fixed with $95 \%$ methanol at room temperature for $15 \mathrm{~min}$, stained with $0.1 \%$ crystal violet at room temperature for $15 \mathrm{~min}$, and captured with the IX71 inverted microscope (Olympus Corporation, Tokyo, Japan). Cell invasion assays were similar to the cell migration assays, except that the Transwell chambers were coated with Matrigel (BD Biosciences). Cell numbers were calculated in five random fields for each Transwell chamber.

Western blot assay. Total proteins were isolated from transfected cells using RIPA buffer containing a protease inhibitor cocktail (Roche, Mannheim, Germany). Concentration of total proteins was quantified with BCA Protein assay (Pierce; Thermo Fisher Scientific). Equal amounts of protein were separated on a $10 \%$ SDS-PAGE and then transferred to polyvinylidene fluoride membranes (PVDF; Bio-Rad Laboratories, Inc., Hercules, CA, USA). Non-specific binding sites of PVDF membranes were saturated with $5 \%$ skimmed milk in TBS 
solution containing $0.1 \%$ Tween 20 (TBST). Subsequently, the membranes were probed with primary antibodies, mouse anti-human monoclonal DLC1 antibody (1:1,000; catalog no. sc-271915; Santa Cruz Biotechnology, Inc., Dallas, TX, USA) and mouse anti-human monoclonal GADPH antibody (1:1,000; catalog no. sc-166574; Santa Cruz Biotechnology), at $4^{\circ} \mathrm{C}$ overnight. After three washes with TBST, the membranes were incubated with corresponding horseradish peroxidase (HRP)-conjugated secondary antibody $(1: 10,000$; Santa Cruz Biotechnology, Inc.) for $2 \mathrm{~h}$ at room temperature, washed with TBST for three times and detected using an enhanced chemiluminescence detection system (ECL; GE Healthcare, Chicago, IL, USA). Relative protein levels of DLC1 were normalized to GAPDH.

Bioinformatic analysis. To predict the potential targets of miR-301a, bioinformatic analysis was performed using TargetScan version $6.2 \mathrm{http} / / /$ www.targetscan.org/vert_71/).

Dual-luciferase reporter assays. The predicted and mutated sequences targeting the 3'UTR of DLC1 were both amplified and cloned into pmirGLO. pmirGLO-DLC1-3'UTR Wt (wild-type) and pmirGLO-DLC1-3'UTR Mut (mutated) were synthesized by Shanghai GenePharma Co., Ltd. 293 cells were seeded in 24-well plates at a density of $1 \times 10^{5}$ cells per well, and co-transfected with miR-301a inhibitor (20 pmol) or NC inhibitor $(20 \mathrm{pmol})$ and pmirGLO-DLC1-3'UTR Wt $(0.2 \mu \mathrm{g})$ or pmirGLO-DLC1-3'UTR Mut $(0.2 \mu \mathrm{g})$ by using Lipofectamine 2000. The transfected cells were harvested, and the relative luciferase activities were determined using Dual-Luciferase Reporter Assay system (Promega Corporation, Madison, WI, USA) $48 \mathrm{~h}$ post-transfection. All experiments were performed in triplicate and repeated three times. Renilla luciferase activity was normalized to firefly luciferase activity.

Statistical analysis. Results were presented as the mean \pm standard deviation. SPSS (version 13.0; SPSS Inc., Chicago, IL, USA) was used for statistical analysis with Student's t-tests or one-way analysis of variance (ANOVA) for comparisons of multiple groups. SNK test was used as a post hoc test following ANOVA. $\mathrm{P}<0.05$ was considered statistically significant.

\section{Results}

miR-301a was upregulated in NSCLC tissues and cell lines. In order to investigate whether miR-301a was differentially expressed in NSCLC, RT-qPCR was performed to analyze miR-301a expression levels in NSCLC tissues and adjacent non-tumor lung tissues. The results indicated that miR-301a was significantly upregulated in NSCLC tissues compared with adjacent non-tumor lung tissues $(\mathrm{P}<0.05$; Fig. 1A).

miR-301a expression in NSCLC cell lines (NCI-H1975, H1299, SPC-A-1, H520 and A549) and a normal human bronchial epithelial cell line (16HBE) was also evaluated. Compared with 16HBE, all five NSCLC cell lines expressed higher levels of miR-301a ( $\mathrm{P}<0.05$; Fig. 1B), which was in accordance with the data from tissue samples $(\mathrm{P}<0.05$; Fig. 1A). The high expression levels of miR-301a in NSCLC tissues and cell lines indicated that miR-301a may act as an oncogene in NSCLC.
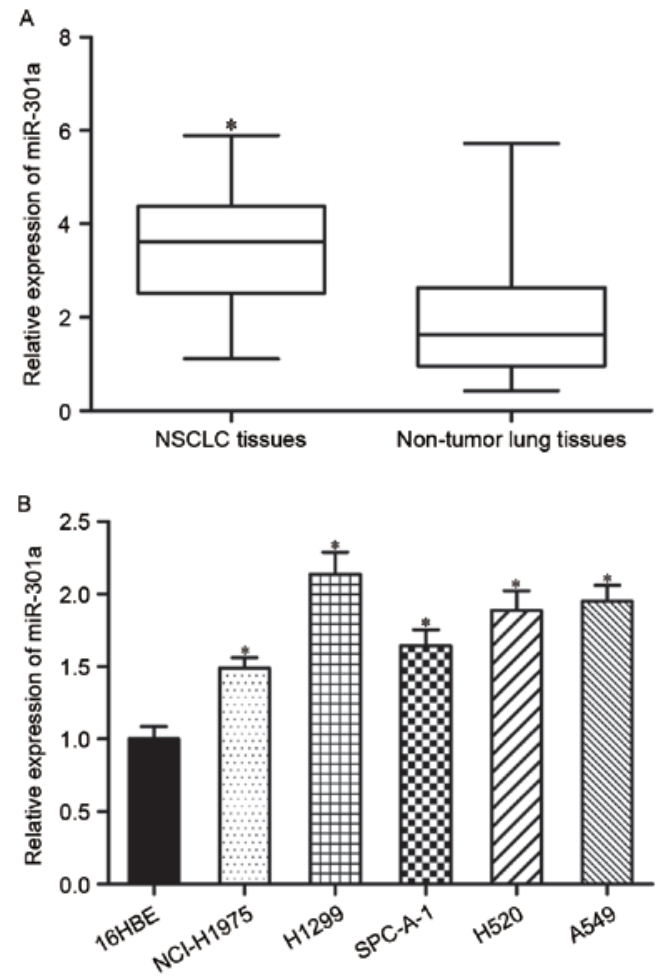

Figure 1. Upregulation of miR-301a in NSCLC tissues and cell lines (A) Relative expression levels of miR-301a in NSCLC tissues and adjacent non-tumor lung tissues. (B) miR-301a expression in five NSCLC cell lines and a normal human bronchial epithelial cell line (16HBE) was analyzed by reverse transcription-quantitative polymerase chain reaction. ${ }^{*} \mathrm{P}<0.05$ vs. 16HBE. NSCLC, non-small cell lung cancer.

Downregulation of miR-30la inhibited proliferation, migration and invasion of NSCLC cells. To investigate the effects of miR-301a on NSCLC progression, H1299 and A549 cells were transfected with miR-301a inhibitor or NC inhibitor. The decrease in the expression of miR-301a in H1299 and A549 cells following transfection with miR-301a inhibitor was confirmed by RT-qPCR (P<0.05; Fig. 2A).

Following transfection with miR-301a inhibitor or NC inhibitor, a series of biological experiments were performed in vitro, including the CCK-8 assay, cell migration and invasion assays. CCK- 8 assay results suggested that miR-301a downregulation significantly inhibited proliferation of H1299 and A549 cells compared with cells transfected with the NC inhibitor $(\mathrm{P}<0.05$; Fig. $2 \mathrm{~B}$ ). In addition, the cell migration assays showed that transfection of the miR-301a inhibitor markedly decreased migratory ability of $\mathrm{H} 1299$ and A549 cells (P<0.05; Fig. 2C). Furthermore, cell invasion assays revealed that the invasive capacity of $\mathrm{H} 1299$ and A549 cells transfected with the miR-301a inhibitor was significantly lower compared with the NC inhibitor groups ( $\mathrm{P}<0.05$; Fig. $2 \mathrm{D}$,). These data demonstrated that downregulation of miR-301a significantly inhibited the proliferation, migration and invasion of NSCLC cells.

DLCl is a target gene of miR-301a in NSCLC. To investigate the molecular mechanisms by which miR-301a downregulation inhibited proliferation, migration and invasion of NSCLC cells, bioinformatic analysis was performed to search for 

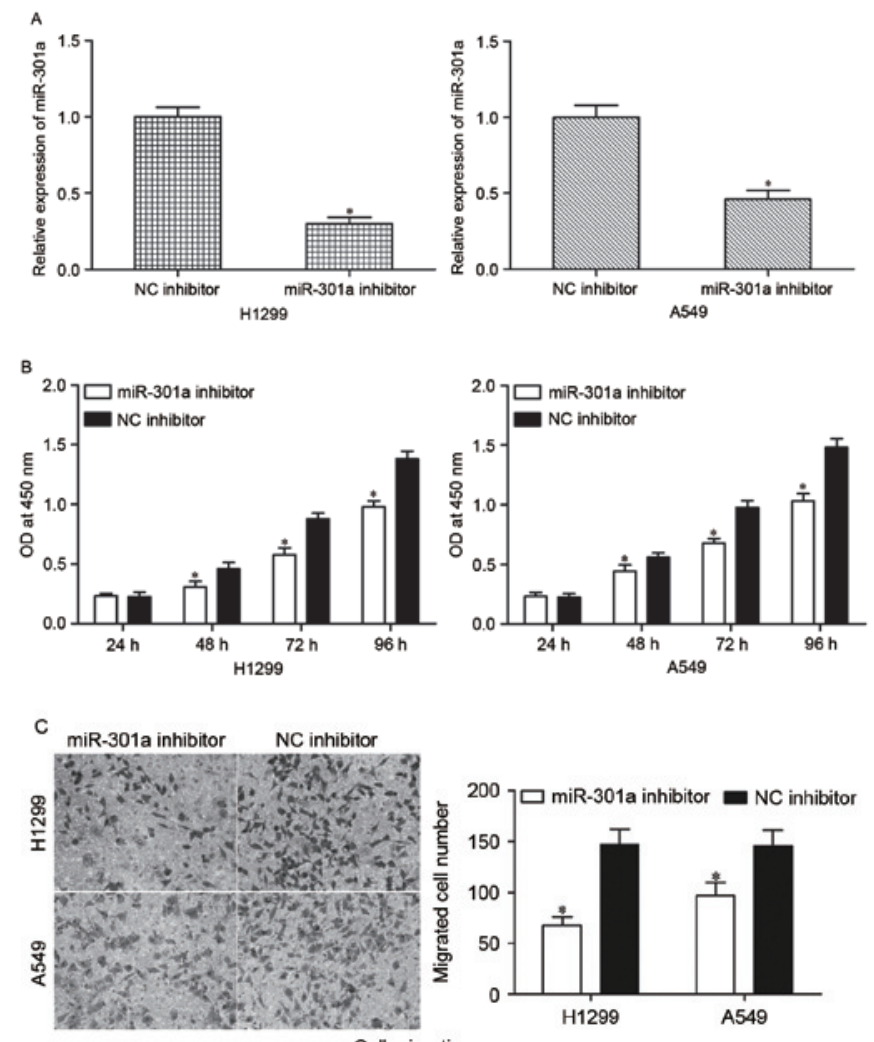

Cell migration assay

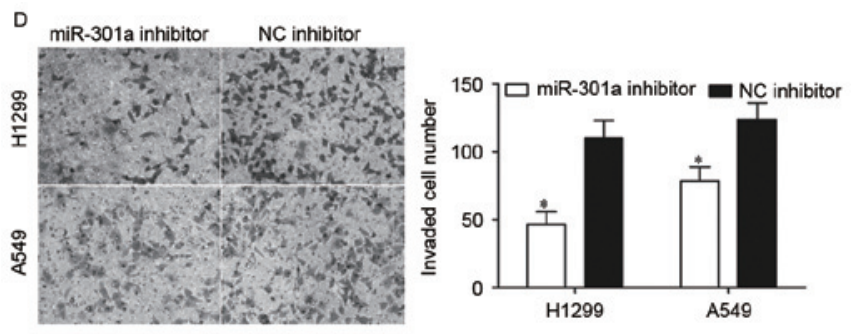

Cell invasion assay

Figure 2. Inhibition of miR-301a represses the proliferation, migration and invasion of H1299 and A549 cells. (A) Expression levels of miR-301a in H1299 and A549 cells that were transfected with miR-301a inhibitor or NC inhibitor were detected by reverse transcription-quantitative polymerase chain reaction. (B) The proliferative ability of the cells was evaluated following transfection with miR-301a inhibitor or NC inhibitor by Cell Counting Kit- 8 assay. The effects of miR-301a inhibitor on (C) migratory and (D) invasive abilities of H1299 and A549 was assessed by cell migration and invasion assays. Magnification, $\mathrm{x} 200 .{ }^{*} \mathrm{P}<0.05$ vs. the NC inhibitor. miRNA, microRNA; NC, negative control; OD, optical density.

potential target genes of miR-301a using TargetScan 6.2. TargetScan 6.2 analysis indicated that DLC1 is a major target of miR-301a (Fig. 3A). DLC1 was chosen for further confirmation because that DLC1 is aberrantly lowly expressed in NSCLC and contributes to NSCLC formation and progression (17-19).

To identify whether DLC1 was a target of miR-301a, dual-luciferase reporter assays were performed. pmirGLO-DLC1-3'UTR Wt or pmirGLO-DLC1-3'UTR Mut, and miR-301a inhibitor or NC inhibitor were co-transfected into 293T cells. As indicated in Fig. 3B, the knockdown of miR-301a increased the luciferase activity compared with cells co-transfected with pmirGLO-DLC1-3'UTR Wt and NC inhibitor. In the cells transfected with pmirGLO-DLC1-3'UTR
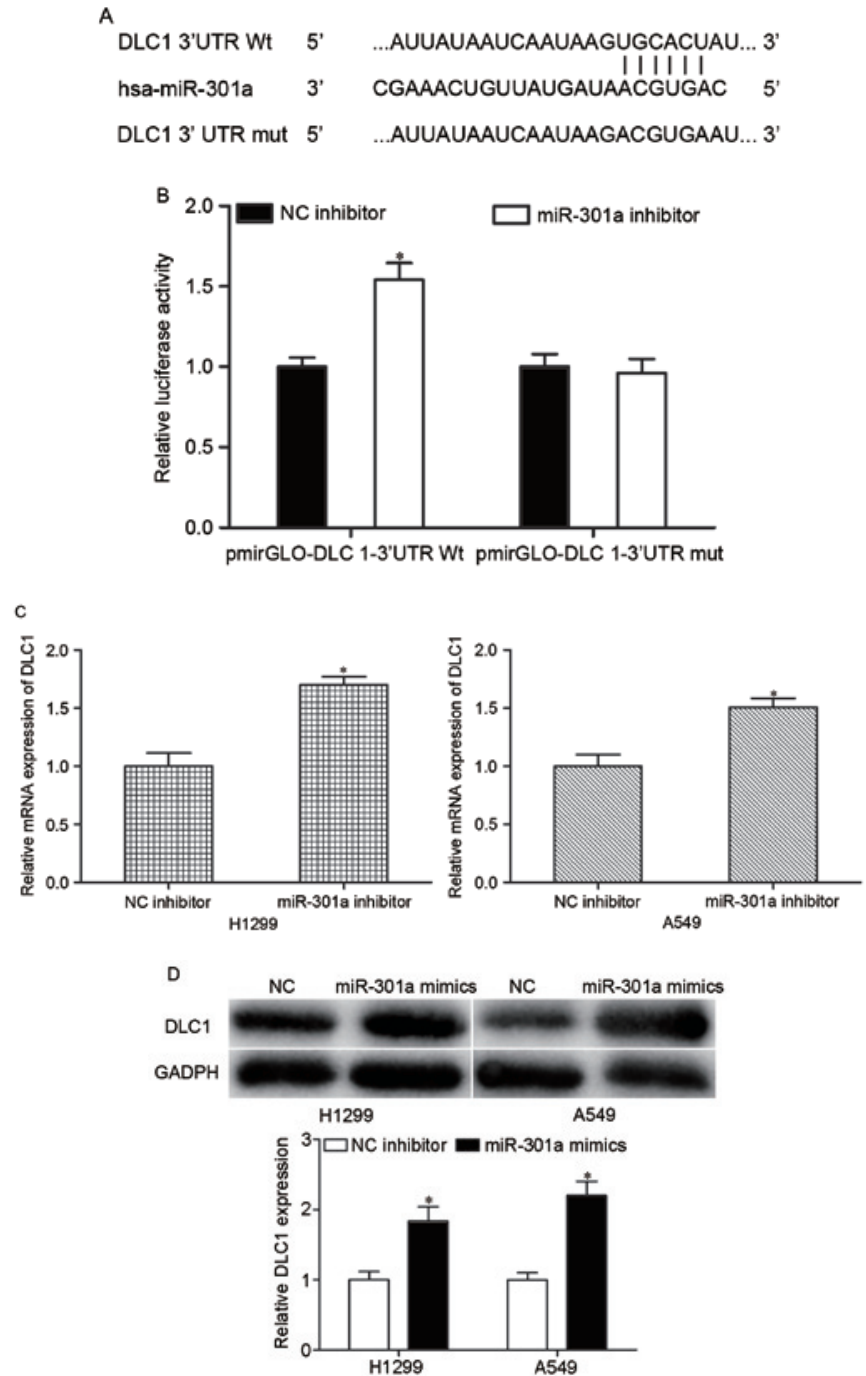

Figure 3. DLC1 is a direct target of miR-301a in NSCLC. (A) Diagram showing the wild-type and mutant binding sites of DLC1-3'UTR for miR-301a. (B) Dual-luciferase reporter assays in 293T cells, following co-transfection of pmirGLO-DLC1-3'UTR Wt or pmirGLO-DLC1-3'UTR Mut and miR-301a inhibitor or NC inhibitor. "P<0.05 vs. NC inhibitor. Reverse transcription-quantitative polymerase chain reaction. And western blot assays indicating the expression levels of (C) DLC1 mRNA and (D) protein in H1299 and A549 cells following transfection with miR-301a inhibitor or NC inhibitor. "P<0.05 vs. NC inhibitor. miRNA, microRNA; mut, mutant wt, wild-type; NC, negative control; UTR, untranslated region.

Mut, there was no significance difference in luciferase activity between cells transfected with NC inhibitor and miR-301a inhibitor $(\mathrm{P}>0.05)$.

Subsequently, RT-qPCR and western blotting was performed to investigate the ability of miR-301a to regulate the expression of DLC1 at mRNA and protein levels, respectively. The results indicated that miR-301a downregulation markedly increased DLC1 expression in H1299 and A549 cells at mRNA (P<0.05; Fig. 3C) and protein $(\mathrm{P}<0.05$; Fig. 3D) levels. Collectively, the findings indicated that DLC1 is a direct target gene of miR-301a in NSCLC.

Knockdown of DLCl partly reverses the effects of miR-301a downregulation on NSCLC cells. To confirm the roles of DLC1 in NSCLC, H1299 and A549 cells were transfected with 
DLC1 siRNA or NC siRNA. The downregulation of DLC1 in H1299 and A549 cells was determined by western blotting ( $\mathrm{P}<0.05$; Fig. 4A).

Next, it was examined whether DLC1 knockdown was able to rescue the carcinogenic effects of miR-301a in NSCLC. The results indicated that downregulation of DLC1 was able to rescue cell proliferation $(\mathrm{P}<0.05$; Fig. $4 \mathrm{~B})$, migratory $(\mathrm{P}<0.05$; Fig. 4C) and invasive $(\mathrm{P}<0.05$; Fig. 4D) abilities of H1299 and A549 cells, which were previously inhibited by miR-301a inhibitor. These findings further verified that DLC1 is a direct target gene of miR-301a and that transfection with miR-301a inhibitor inhibited proliferation, migration and invasion of NSCLC cells, at least in part by inducing expression of DLC1.

\section{Discussion}

NSCLC is one of the most common malignant diseases with a high mortality rate (20). NSCLC carcinogenesis is a multi-stage process, which involves a broad spectrum of changes in gene expression and physiological structure (21). The abnormal expression of genes mainly includes activation of oncogenes and inactivation of tumor suppressor genes (22). Accumulated evidence have suggested that miRNA is involved in carcinogenesis as either oncogene or tumor suppressor and the functions of many cancer-associated miRNAs have been identified $(23,24)$. Moreover, dysregulation of miRNAs expression has been indicated to be potential sensitive and accurate biomarkers for cancer diagnosis and prognosis of human cancer $(25,26)$. Therefore, understanding the roles of miRNAs in NSCLC might provide valuable information for therapeutic strategies in the therapy for patients with NSCLC. The present study focused on the roles of miR-301a in NSCLC. The present study investigated the expression and biological functions of miR-301a in NSCLC as well as the potential mechanism by which miR-301a acts as an oncogene in NSCLC initiation and progression. The findings indicated that: i) miR-301a was significantly upregulated in NSCLC tissues and cell lines compared with adjacent non-tumor lung tissues and normal human bronchial epithelial cell line, respectively; ii) miR-301a downregulation inhibited the proliferation, migration and invasion of NSCLC cells; iii) bioinformatic analysis showed that DLC1 is a putative target of miR-301a; iv) miR-301a downregulation increased the expression levels of DLC1 by directly targeting its 3'UTR; v) DLC1 knockdown partially reverses the inhibition of proliferation, migration and invasion induced by downregulation of miR-301a in NSCLC cells.

Previous studies have shown that miR-301a was closely associated with multiple malignant phenotypes of human cancer. miR-301a was reported to be upregulated in numerous types of human cancer, including Ewing's sarcoma (27), laryngeal squamous cell carcinoma (28), pancreatic cancer (29), colorectal cancer (30), gastric cancer (31) and hepatocellular carcinoma (32). Furthermore, the levels of miR-301a expression were demonstrated to be correlated with clinicopathological features in cancer patients. Xu et al (31) reported that in gastric cancer, miR-301a was associated with tumor size, invasion depth, lymph node metastasis and tumor-node-metastasis (TNM) stage. Yu et al (33) reported that the expression level of miR-301a in triple-negative breast cancer was positively correlated with tumor size, depth of invasion, TNM stage
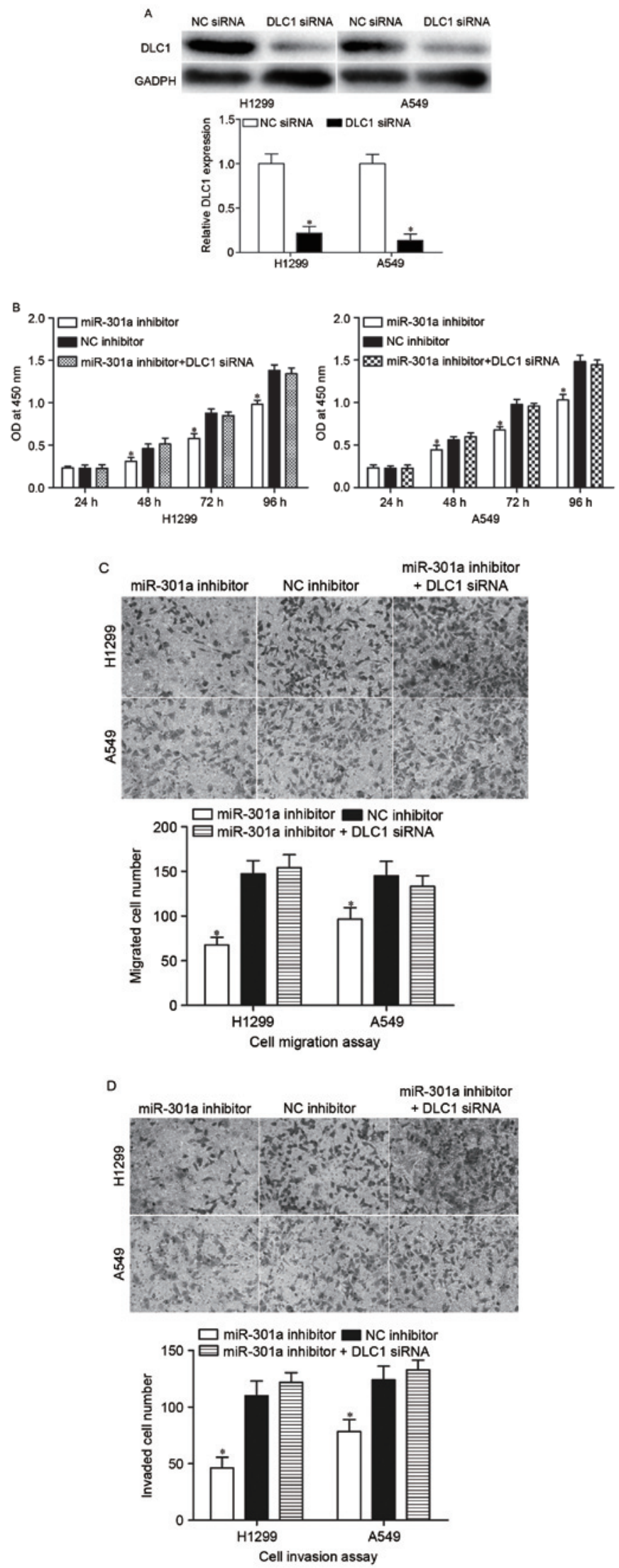

Figure 4. Knockdown of DLC1 reverses the effects of miR-301a downregulation in NSCLC cells. (A) Expression levels of DLC1 in H1299 and A549 cells transfected with DLC1 siRNA or NC siRNA were analyzed by western blotting. Knockdown of DLC1 markedly reversed the carcinogenic effects of miR-301a on (B) proliferation, (C) migration and (D) invasion of NSCLC cells. Magnification, $x 200 .^{*} \mathrm{P}<0.05$ vs. the NC inhibitor. miRNA, microRNA; NC, negative control; NSCLC, non-small cell lung cancer; siRNA, small-interfering RNA. 
and lymph node metastasis. In addition, multivariate analysis suggested that miR-301a expression was an independent prognostic factor for the survival of patients with triple-negative breast cancer (33). Xia and colleagues (29) showed that higher expression of miR-301a was observed in pancreatic cancer patients with lymph node metastasis and advanced pathological stages. miR-301a was also identified as an independent prognostic factor for worse survival (29). These findings suggested that miR-301a was upregulated in various types of cancer and may act as diagnostic and prognostic biomarkers.

Furthermore, studies have also revealed that miR-301a is able to act as an oncogene in carcinogenesis and progression of human cancer. For example, in Ewing's sarcoma, knockdown of miR-301a suppressed proliferation and cell cycle progression (27). Furthermore, downregulation of miR-301a in Ewing's sarcoma cells significantly suppressed tumor growth in vivo (27). Xia et al indicated that upregulation of miR-301a promoted pancreatic cancer cell colony formation, invasive and migratory abilities in vitro as well as tumorigenicity in vivo (29). Fang et al found that miR-301a acts as an oncogene in colorectal cancer by increasing proliferation, migration and invasion as well as tumor growth (30). In breast cancer, exogenous expression of miR-301a expression increased cell migration, invasion and metastasis both in vitro and in vivo (34). Zhou et al (32) showed that miR-301a increased proliferation, migration and invasion of hepatocellular carcinoma cells and inhibited apoptosis. In accordance with these results, the findings of the present study indicated that inhibition of miR-301a expression decreased proliferation, migration and invasion of NSCLC cells. These findings suggested that miR-301a may be a therapeutic target in NSCLC.

Previously, a number of predicted targets of miR-301a have been reported in various types of cancer, including GAX in hepatocellular carcinoma (32), BIM (35) and SMAD family member 4 (29) in pancreatic cancer, phosphatase and tensin homolog (34) and runt related transcription factor 3 (36) in gastric cancer, TGFBR2 (37) and suppressor of cytokine signaling 6 (30) in colorectal cancer and AMPK $\alpha 1$ in osteosarcoma (38).

To investigate the molecular mechanism by which miR-301a contributes to proliferation, migration and invasion of NSCLC, the potential target genes of miR-301a were investigated. In the present study, DLC1 was identified as a novel target of miR-301a. Initially, bioinformatic analysis indicated that DLC1 was a putative target of miR-301a. Next, dual-luciferase reporter assays showed that miR-301a directly targeted the 3'UTR of DLC1. Downregulation of miR-301a increased DLC1 expression at mRNA and protein levels. DLC1 knockdown also partially reversed the inhibition of proliferation, migration and invasion induced by miR-301a knockdown in NSCLC cells. All these results indicated that DLC1 is a direct target gene of miR-301a in NSCLC. Identification of target genes of miR-301a is important for elucidating the functions of miR-301a in carcinogenesis and progression of NSCLC, which may provide promising therapeutic targets for NSCLC.

In conclusion, the present study demonstrated that miR-301a was upregulated in NSCLC tissues and cell lines compared with normal adjacent tissues and a normal human bronchial epithelial cell line. Functional analyses indicated that miR-301a may promote proliferation, migration and invasion of NSCLC cells by directly targeting DLC1. Therefore, miR-301a may contribute to the initiation and progression of NSCLC. These findings may help to further elucidate the molecular mechanisms underlying the carcinogenesis and progression of NSCLC, and provide evidence for the miR-301a/DLC1 pathway as a potential therapeutic target for patients with NSCLC.

\section{References}

1. Siegel R, Naishadham D and Jemal A: Cancer statistics, 2012. CA Cancer J Clin 62: 10-29, 2012.

2. Spira A and Ettinger DS: Multidisciplinary management of lung cancer. N Engl J Med 350: 379-392, 2004.

3. Yang L, Parkin DM, Li L and Chen Y: Time trends in cancer mortality in China: 1987-1999. Int J Cancer 106: 771-783, 2003.

4. Pfister DG, Johnson DH, Azzoli CG, Sause W, Smith TJ, Baker S Jr, Olak J, Stover D, Strawn JR, Turrisi AT, et al: American Society of Clinical Oncology treatment of unresectable non-small-cell lung cancer guideline: Update 2003. J Clin Oncol 22: 330-353, 2004

5. Yilmaz A, Damadoglu E, Salturk C, Okur E, Tuncer LY and Halezeroglu S: Delays in the diagnosis and treatment of primary lung cancer: Are longer delays associated with advanced pathological stage? Ups J Med Sci 113: 287-296, 2008.

6. Verdecchia A, Francisci S, Brenner H, Gatta G, Micheli A, Mangone L and Kunkler I; EUROCARE-4 Working Group: Recent cancer survival in Europe: A 2000-02 period analysis of EUROCARE-4 data. Lancet Oncol 8: 784-796, 2007.

7. Somaiah N and Simon GR: Molecular targeted agents and biologic therapies for non-small cell lung cancer. J Thorac Oncol 5 (12 Suppl 6): S434-S454, 2010.

8. Bartel DP: MicroRNAs: Genomics, biogenesis, mechanism, and function. Cell 116: 281-297, 2004.

9. Calin GA and Croce CM: MicroRNA signatures in human cancers. Nat Rev Cancer 6: 857-866, 2006.

10. Lewis BP, Burge CB and Bartel DP: Conserved seed pairing, often flanked by adenosines, indicates that thousands of human genes are microRNA targets. Cell 120: 15-20, 2005.

11. Donadeu FX, Schauer SN and Sontakke SD: Involvement of miRNAs in ovarian follicular and luteal development. J Endocrinol 215: 323-334, 2012.

12. Liwak U, Faye MD and Holcik M: Translation control in apoptosis. Exp Oncol 34: 218-230, 2012.

13. Rutnam ZJ and Yang BB: The involvement of microRNAs in malignant transformation. Histol Histopathol 27: 1263-1270, 2012 .

14. Rothschild SI: Epigenetic therapy in lung cancer-role of microRNAs. Front Oncol 3: 158, 2013.

15. Volinia S, Calin GA, Liu CG, Ambs S, Cimmino A, Petrocca F, Visone R, Iorio M, Roldo C, Ferracin M, et al: A microRNA expression signature of human solid tumors defines cancer gene targets. Proc Natl Acad Sci USA 103: 2257-2261, 2006.

16. Livak KJ and Schmittgen TD: Analysis of relative gene expression data using real-time quantitative PCR and the 2(-Delta Delta C(T)) method. Methods 25: 402-408, 2001.

17. Yuan BZ, Jefferson AM, Baldwin KT, Thorgeirsson SS, Popescu NC and Reynolds SH: DLC-1 operates as a tumor suppressor gene in human non-small cell lung carcinomas. Oncogene 23: 1405-1411, 2004

18. Feng H, Zhang Z, Wang X and Liu D: Identification of DLC-1 expression and methylation status in patients with non-small-cell lung cancer. Mol Clin Oncol 4: 249-254, 2016.

19. Healy KD, Hodgson L, Kim TY, Shutes A, Maddileti S, Juliano RL, Hahn KM, Harden TK, Bang YJ and Der CJ: DLC-1 suppresses non-small cell lung cancer growth and invasion by RhoGAP-dependent and independent mechanisms. Mol Carcinog 47: 326-337, 2008.

20. Liu XH, Liu ZL, Sun M, Liu J, Wang ZX and De W: The long non-coding RNA HOTAIR indicates a poor prognosis and promotes metastasis in non-small cell lung cancer. BMC Cancer 13: 464, 2013.

21. Chen X, Chen S, Hang W, Huang H and Ma H: MiR-95 induces proliferation and chemo- or radioresistance through directly targeting sorting nexin1 (SNX1) in non-small cell lung cancer. Biomed Pharmacother 68: 589-595, 2014.

22. Zhang Y, Zhao Y, Sun S, Liu Z, Zhang Y and Jiao S: Overexpression of MicroRNA-221 is associated with poor prognosis in non-small cell lung cancer patients. Tumour Biol 37: 10155-10160, 2016. 
23. Chen X, Gong J, Zeng H, Chen N, Huang R, Huang Y, Nie L, $\mathrm{Xu} \mathrm{M}$, Xia J, Zhao F, et al: MicroRNA145 targets BNIP3 and suppresses prostate cancer progression. Cancer Res 70: 2728-2738, 2010.

24. Wu D, Zhou Y, Pan H, Zhou J, Fan Y and Qu P: microRNA-99a inhibiting cell proliferation, migration and invasion by targeting fibroblast growth factor receptor 3 in bladder cancer. Oncol Lett 7: 1219-1224, 2014.

25. Xu T, Liu X, Han L, Shen H, Liu L and Shu Y: Up-regulation of miR-9 expression as a poor prognostic biomarker in patients with non-small cell lung cancer. Clin Transl Oncol 16: 469-475, 2014.

26. Bai Y, Wang YL, Yao WJ, Guo L, Xi HF, Li SY and Zhao BS: Expression of miR-32 in human non-small cell lung cancer and its correlation with tumor progression and patient survival. Int J Clin Exp Pathol 8: 824-829, 2015.

27. Kawano M, Tanaka K, Itonaga I, Iwasaki $\mathrm{T}$ and Tsumura $\mathrm{H}$ : MicroRNA-301a promotes cell proliferation via PTEN targeting in Ewing's sarcoma cells. Int J Oncol 48: 1531-1540, 2016.

28. Lu Y, Gao W, Zhang C, Wen S, Huangfu H, Kang J and Wang B: Hsa-miR-301a-3p acts as an oncogene in laryngeal squamous cell carcinoma via target regulation of Smad4. J Cancer 6: 1260-1275, 2015.

29. Xia X, Zhang K, Cen G, Jiang T, Cao J, Huang K, Huang C, Zhao Q and Qiu Z: MicroRNA-301a-3p promotes pancreatic cancer progression via negative regulation of SMAD4. Oncotarget 6: 21046-21063, 2015.

30. Fang Y, Sun B, Xiang J and Chen Z: MiR-301a promotes colorectal cancer cell growth and invasion by directly targeting SOCS6. Cell Physiol Biochem 35: 227-236, 2015.
31. Xu XD, He XJ, Tao HQ, Zhang W, Wang YY, Ye ZY and Zhao ZS: Abnormal expression of miR-30la in gastric cancer associated with progression and poor prognosis. J Surg Oncol 108: 197-202, 2013.

32. Zhou P, Jiang W, Wu L, Chang R, Wu K and Wang Z: miR-301a is a candidate oncogene that targets the homeobox gene Gax in human hepatocellular carcinoma. Dig Dis Sci 57: 1171-1180, 2012.

33. Yu H, Li H, Qian H, Jiao X, Zhu X, Jiang X, Dai G and Huang J: Upregulation of miR-301a correlates with poor prognosis in triple-negative breast cancer. Med Oncol 31: 283, 2014.

34. Ma F, Zhang J, Zhong L, Wang L, Liu Y, Wang Y, Peng L and Guo B: Upregulated microRNA-301a in breast cancer promotes tumor metastasis by targeting PTEN and activating Wnt/ $\beta$-catenin signaling. Gene 535: 191-197, 2014.

35. Chen Z, Chen LY, Dai HY, Wang P, Gao S and Wang K: miR-301a promotes pancreatic cancer cell proliferation by directly inhibiting Bim expression. J Cell Biochem 113: 3229-3235, 2012.

36. Wang M, Li C, Yu B, Su L, Li J, Ju J, Yu Y, Gu Q, Zhu Z and Liu B: Overexpressed miR-301a promotes cell proliferation and invasion by targeting RUNX3 in gastric cancer. J Gastroenterol 48: 1023-1033, 2013.

37. Zhang W, Zhang T, Jin R, Zhao H, Hu J, Feng B, Zang L, Zheng M and Wang M: MicroRNA-301a promotes migration and invasion by targeting TGFBR2 in human colorectal cancer. J Exp Clin Cancer Res 33: 113, 2014.

38. Zhang Y, Duan G and Feng S: MicroRNA-301a modulates doxorubicin resistance in osteosarcoma cells by targeting AMP-activated protein kinase alpha 1. Biochem Biophys Res Commun 459: 367-373, 2015. 